\title{
Chapter 18 \\ Functional Roles of spe Genes in the Male \\ Germline During Reproduction of Caenorhabditis elegans
}

\author{
Hitoshi Nishimura, Tatsuya Tajima, Skye Comstra, \\ and Steven W. L'Hernault
}

\begin{abstract}
The nematode Caenorhabditis elegans is an excellent model animal to study various biological phenomena, including reproduction. In this chapter, we focus on functional roles of spermatogenesis- or sperm-defective (spe) genes in the C. elegans male germline during reproduction. So far, approximately 190 mutants of $C$. elegans that are defective in male germline functions have been isolated, and many of them carry mutated alleles for one of the perhaps 60 spe genes. Most spe genes exhibit male germline-specific expression and play roles during spermatogenesis (spermatid production), spermiogenesis (spermatid activation into sperm), or fertilization. For example, spe- 8 class genes are indispensable for hermaphroditedependent spermiogenesis. If either of the spe- 8 class genes is aberrant, spermatids from mutant hermaphrodites, but not from males, arrest at an intermediate stage during spermiogenesis. In contrast, fertilization requires spe-9 class genes. Hermaphrodites and males of spe-9 class mutants produce otherwise normal sperm that are incapable of fertilizing oocytes. Because $C$. elegans oocytes have no egg coats, spe- 9 class genes are probably required for sperm to bind to and/or fuse with the oocyte plasma membrane. Intriguingly, several spe genes are likely to be orthologues of mammalian genes, suggesting that $C$. elegans and mammals share some common steps during male germline functions at the molecular level.
\end{abstract}

\footnotetext{
H. Nishimura $(\triangle)$

Department of Life Science, Setsunan University, 17-8 Ikeda-Nakamachi,

Neyagawa, Osaka 572-8508, Japan

Department of Biology, Emory University, 1510 Clifton Road NE, Atlanta, GA 30322, USA

e-mail: nishimura@ lif.setsunan.ac.jp

T. Tajima

Department of Life Science, Setsunan University, 17-8 Ikeda-Nakamachi,

Neyagawa, Osaka 572-8508, Japan

S. Comstra • S.W. L'Hernault

Department of Biology, Emory University, 1510 Clifton Road NE, Atlanta, GA 30322, USA
} 
Keywords Caenorhabditis elegans $•$ Fertilization • Male germline $\bullet$ spe genes - Spermiogenesis

\subsection{Overview of Caenorhabditis elegans Reproduction}

In many life science research fields, the nematode Caenorhabditis elegans is utilized as a model animal. The advantage in using this worm is that we can observe biological phenomena in vivo and easily manipulate its genes, about $40 \%$ of which are shared with those of other animal species (C. elegans Sequencing Consortium 1998). In particular, the many C. elegans mutants available are so powerful in investigating reproduction because sperm and oocytes are haploid, cell types that are too specialized to examine gamete-specific gene functions in vitro.

C. elegans somatic cells have five pairs of autosomes (I, II, III, IV, V) and one pair of sex chromosomes (XX or XO). Therefore, there are two sexes in C. elegans, an XX hermaphrodite and an XO male (Fig. 18.1).

In a hermaphrodite, spermatogenesis occurs in both arms of the U-shaped gonad during the fourth larval (L4) stage. As L4 hermaphrodites become young adults, spermatogenesis completely switches to oogenesis. Hence, adult hermaphrodites are somatically females, despite the presence of self-sperm in the spermatheca.
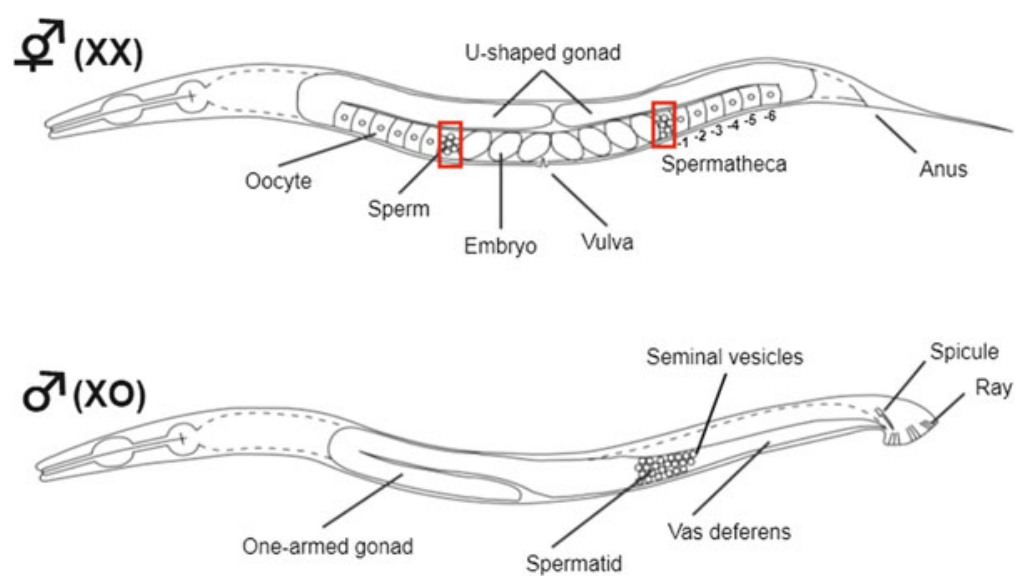

Fig. 18.1 Hermaphrodite and male of the nematode Caenorhabditis elegans. Top: An adult hermaphrodite (sex genotype, XX) has a U-shaped gonad that exhibits mirror image symmetry around the single vulva. Location of each spermatheca is outlined by squares. Numbers below the gonad indicate positions of oocytes according to their developmental stages. Bottom: An adult male (XO) has a one-armed gonad. Spermatids accumulate in the single vas deferens until they are ejaculated during mating to a hermaphrodite. (These figures were taken from the article by Nishimura and L'Hernault 2010 with the publisher's permission) 


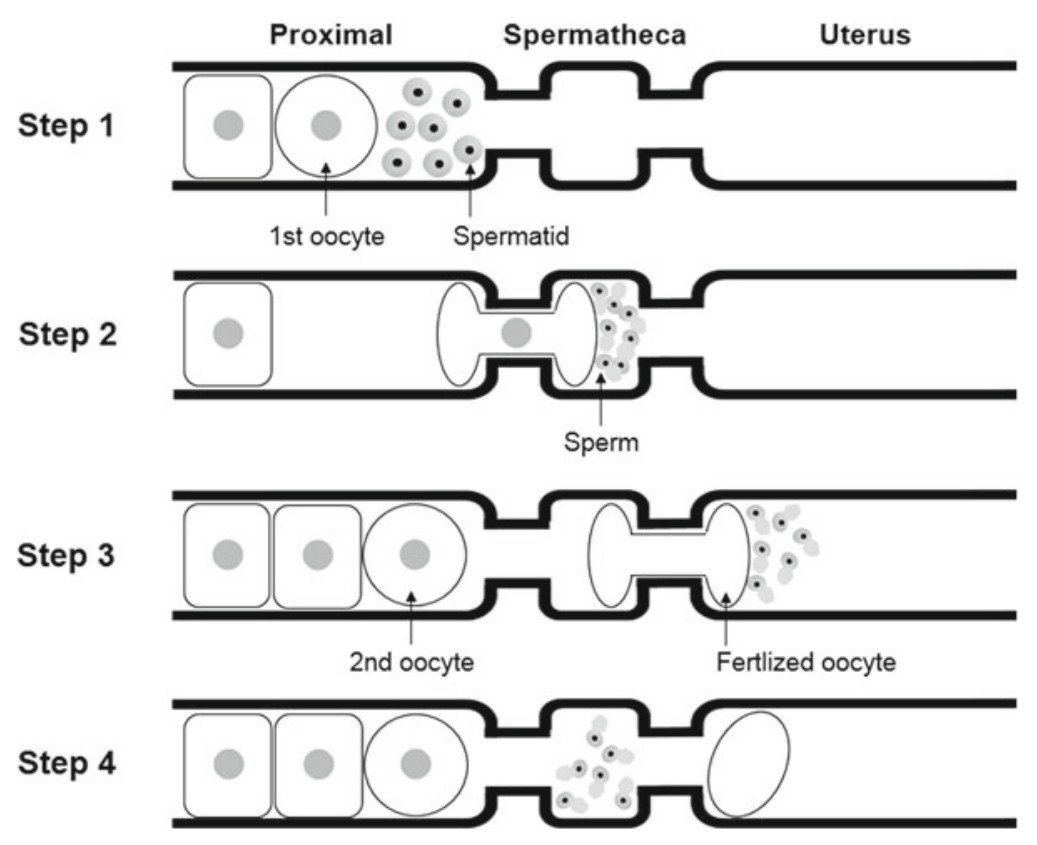

Fig. 18.2 Ovulation-fertilization cycle in the gonad of C. elegans hermaphrodite. Each figure shows an expanded area of the hermaphroditic gonad, including the proximal gonad, spermatheca, and uterus. For details, see Sect. 18.1

Oogenesis is ongoing from the distal to the proximal direction in the adult worm gonads, so that the fertilization-ready oocyte resides at the most proximal region ( -1 position in Fig. 18.1). Figure 18.2 shows a scheme of the ovulation-fertilization cycle. Before the first ovulation, self-spermatids that had been produced during the L4 stage are localized in the proximal gonads of adult hermaphrodites (Step 1). When the first -1 oocyte is ovulated, it pushes the previously produced spermatids out of the proximal gonad into the spermatheca (Step 2). Those spermatids rapidly transform into sperm (self-sperm) in the spermatheca, and one of them fertilizes the first oocyte. Then, together with the remaining sperm, the fertilized oocyte moves into the uterus for the onset of embryogenesis (Step 3). The uterine sperm crawl back into the spermatheca and compete again for the next fertilization (Step 4). An adult hermaphrodite carries approximately 300 self-sperm in the spermatheca and eventually produces about 300 self-progeny. Thus, although the self-sperm are pushed into the uterus at every ovulation, most are capable of crawling back into the spermatheca so that nearly all the sperm can be consumed by fertilization. When self-fertilization occurs in wild-type hermaphrodites, $99.9 \%$ of self-progeny are XX hermaphrodites.

In a male (Fig. 18.1), spermatogenesis first occurs at the L4 stage and lasts throughout the entire life of the animal. Wild-type male spermatids are barely activated into sperm in the gonad. During mating to a hermaphrodite, male spermatids are ejaculated into the uterus through the vulva and then activated into sperm. The male sperm 
subsequently crawl into the spermatheca to fertilize oocytes. If self-sperm already exist in the spermatheca, male sperm have an advantage to fertilize oocytes, probably because of the larger size and faster crawling velocity of male-derived sperm than those of self-sperm. Consequently, after mating, fertilization mostly occurs between oocytes and male sperm, rather than between oocytes and self-sperm. This observation also indicates that outcrossing of an XX hermaphrodite and an XO male results in about half the progeny being males, in contrast to self-fertilization.

\subsection{Male Germline Functions in C. elegans}

\subsubsection{Spermatogenesis}

As shown in Fig. 18.3, during C. elegans reproduction, the male germline is involved in spermatogenesis, spermiogenesis, and fertilization (Singson et al. 2008; L'Hernault 2009; Nishimura and L'Hernault 2010). Spermatogenesis is a process that produces four haploid spermatids from one diploid spermatocyte via meiosis (Fig. 18.3a). A primary spermatocyte is first divided into two secondary

a Spermatogenesis

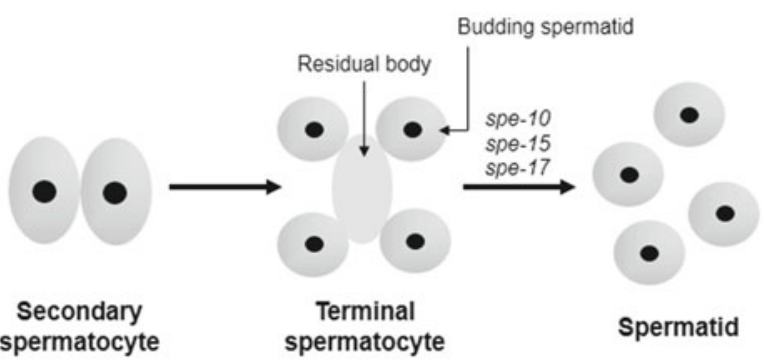

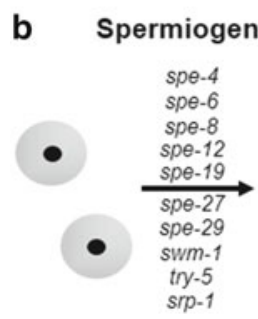

Spermatid

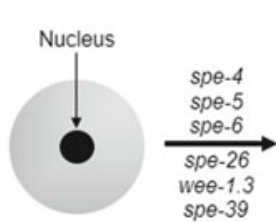

Primary spermatocyte spermatocyte

c

Fertilization

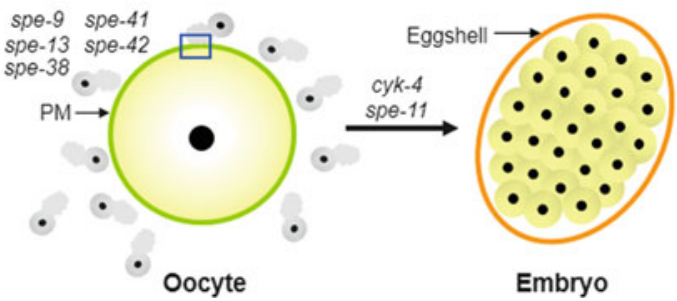

Fig. 18.3 C. elegans spe genes acting during male germline functions. A part of the spe genes that are involved in either of spermatogenesis (a), spermiogenesis (b), or fertilization (c) are shown in these figures. In c, a square shows that a sperm contacts the oocyte plasma membrane through its pseudopod. (These figures were taken from the article by Nishimura and L'Hernault 2010 with the publisher's permission) 
spermatocytes during meiosis I. Then, each secondary spermatocyte undergoes meiosis II to generate two haploid spermatids, which have budded from an acellular residual body. The second cell division is asymmetrical, and the residual body receives many organelles and cytoplasmic proteins. One of the cytological features of mature spermatids is that this cell type possesses Golgi-derived, secretory membranous organelles (MOs) in the cytoplasm.

In contrast to mammals, $C$. elegans spermatogenesis is readily reproducible in vitro; spermatocytes that are released from dissected males can differentiate into spermatids in a simple, chemically defined medium (L'Hernault 2009; Nishimura and L'Hernault 2010). Because this in vitro spermatogenesis can be completed within approximately $90 \mathrm{~min}$, use of $C$. elegans provides a significant advantage in studying spermatogenesis.

\subsubsection{Spermiogenesis}

During $C$. elegans spermiogenesis, a sessile round spermatid transforms into a motile, amoeboid sperm (Fig. 18.3b). Because no ribosomes are present in spermatids, spermiogenesis does not entail de novo protein synthesis. For crawling, each C. elegans sperm has a single pseudopod extending from its cell body, instead of a flagellum. The pseudopod also acts as a binding/fusion site with the oocyte plasma membrane (Fig. 18.3c). Nematode sperm, differing from other amoeboid-like cell types, utilize the major sperm protein (MSP) as their cytoskeletal protein.

The MOs, which are present in the cytoplasm of a spermatid, have a secretory function during spermiogenesis. The MOs fuse with the spermatid plasma membrane and release their contents extracellularly. Moreover, some sperm proteins that play essential roles in fertilization translocate from the MOs to the pseudopod surface (see Sect. 18.3.3). In this aspect, the MO might be analogous to the acrosome of flagellated sperm.

The seminal fluid contains the serine protease TRY-5, and this protein probably activates male-derived spermatids in vivo (Smith and Stanfield 2011) (see Sect. 18.3.2.2). TRY-5 might also activate hermaphrodite-derived spermatids, but hermaphrodites likely have a unknown, physiological activator(s) besides TRY-5. In vitro, spermatids can be activated into sperm by treatment with either the cationic ionophore Monensin, the weak base triethanolamine (TEA), the phosphoinositide3-kinase inhibitor Wortmannin, or the bacterial serine protease mixture Pronase. Sperm generated by in vitro activation with TEA, but not Pronase, are competent to fertilize oocytes after the sperm are artificially inseminated into hermaphrodites.

\subsubsection{Fertilization}

C. elegans fertilization (Fig. 18.3c) is distinguished from mammalian fertilization in several ways. First, mammalian sperm undergo the acrosome reaction, which is essentially required before sperm-oocyte fusion. In contrast, C. elegans sperm lack 
an acrosome, probably because of the absence of any substantial egg coat (ex. the zona pellucida) surrounding C. elegans oocytes. Second, acrosome-reacted sperm of mammals bind to the oocyte plasma membrane at the equatorial region of the sperm head, whereas $C$. elegans sperm probably first contact the oocyte plasma membrane via the pseudopod (Fig. 18.3c), which functionally corresponds to the flagella of mammalian sperm. Third, in contrast to mammals, a system of in vitro fertilization (IVF) is not yet available for C. elegans.

On the other hand, the reproductive tract of $C$. elegans adult hermaphrodites is functionally analogous to that of mammalian females; the proximal gonad, spermatheca, and uterus of adult hermaphrodites play similar spatial roles to those of the ovary, oviduct, and uterus of mammals. As described in Sect. 18.1, adult hermaphrodites are somatically females and no longer produce self-sperm. Hence, C. elegans might provide valuable insights into certain aspects of in vivo fertilization that are analogous to mammalian fertilization.

\section{3 spe Genes Acting During the C.elegans Male Germline Functions}

\subsubsection{What Are spe Genes?}

Mutants lacking either type of spe (spermatogenesis- or sperm-defective) genes produce spermatocytes, spermatids, or sperm of which the functions are aberrant during spermatogenesis, spermiogenesis, and fertilization (Singson et al. 2008; L'Hernault 2009; Nishimura and L'Hernault 2010) (Fig. 18.3). spe mutant hermaphrodites produce very few progeny and instead lay unfertilized oocytes. However, mating to wild-type males allows spe mutant hermaphrodites to produce outcross progeny. Thus, sperm, but not oocytes, are functionally defective in spe mutants. In other words, wild-type sperm are necessary and sufficient to rescue the self-sterility of mutant hermaphrodites. Using these criteria, mutants that define about 60 spe genes have been isolated after treatment of hermaphrodites with chemical mutagens.

Most spe genes are expressed specifically or predominantly in the C. elegans male germline as expected, although some genes that play important roles in male germline functions are also expressed in other tissues. Among spe genes so far identified, spe- 8 class and spe- 9 class genes have been intensively studied; spe- 8 class genes (spe-8, spe-12, spe-19, spe-27, and spe-29) act in hermaphrodite-dependent spermiogenesis (see Sect. 18.3.2.1 and Table 18.1), whereas fertilization requires spe-9 class genes (spe-9, spe-13, spe-38, spe-41/trp-3, and spe-42) (see Sect. 18.3.3 and Table 18.2). 
Table 18.1 spe genes involved in spermiogenesis

\begin{tabular}{|c|c|c|c|}
\hline Gene & Chr. & Predicted protein & Protein localization \\
\hline$\overline{\text { spe-4 }}$ & I & $\begin{array}{l}\text { Seven-pass transmembrane aspartyl protease } \\
\text { of the presenilin family (465 aa) }\end{array}$ & $\begin{array}{l}\text { MOs of spermatid } \\
\text { Cell body of sperm }\end{array}$ \\
\hline spe-6 & III & $\begin{array}{l}\text { Ser/Thr kinase of the casein kinase } 1 \text { family } \\
\text { (379 aa) }\end{array}$ & ND \\
\hline spe-8 & I & $\begin{array}{l}\text { Nonreceptor type of tyrosine kinase with the } \mathrm{SH} 2 \\
\text { domain }(512 \mathrm{aa})\end{array}$ & ND \\
\hline spe-12 & I & Transmembrane protein (255 aa) & ND \\
\hline spe-19 & V & $\begin{array}{l}\text { Transmembrane protein with } 11 \text { potential } \\
\text { phosphorylation sites in the cytoplasmic } \\
\text { tail }(300 \mathrm{aa})\end{array}$ & ND \\
\hline spe-27 & IV & Soluble protein $(131 \mathrm{aa})$ & ND \\
\hline spe-29 & IV & Transmembrane protein (66 aa) & ND \\
\hline try-5 & $\mathrm{V}$ & Soluble trypsin-like protease (327 aa) & Seminal fluid \\
\hline swm-1 & V & $\begin{array}{l}\text { Soluble protein with two trypsin inhibitor-like } \\
\text { domains ( } 86 \text { and } 135 \mathrm{aa})\end{array}$ & Likely seminal fluid \\
\hline srp-1 & V & $\begin{array}{l}\text { Ovalbumin-like protein of the Serpin } \\
\text { (serine protease inhibitor) family ( } 366 \text { aa) }\end{array}$ & $\begin{array}{l}\text { MOs of spermatid } \\
\text { (Ce and As) } \\
\text { Cell body and pseudopod } \\
\text { of sperm (As) }{ }^{\mathrm{a}}\end{array}$ \\
\hline
\end{tabular}

Table 18.2 spe genes involved in fertilization

\begin{tabular}{|c|c|c|c|}
\hline Gene & Chr. & Predicted protein & Protein localization \\
\hline spe-9 & I & $\begin{array}{l}\text { Transmembrane protein with ten EGF-like domains, } \\
\text { similar to Delta, Serrate, and Jagged proteins } \\
\text { (381 and/or } 661 \text { aa) }\end{array}$ & $\begin{array}{l}\text { MOs of spermatid } \\
\text { Pseudopod of sperm }\end{array}$ \\
\hline spe-13 & I & ND & ND \\
\hline spe-38 & I & Four-pass transmembrane protein (179 aa) & $\begin{array}{l}\text { MOs of spermatid } \\
\text { Pseudopod of sperm }\end{array}$ \\
\hline spe-41(trp-3) & III & $\begin{array}{l}\mathrm{Ca}^{2+} \text {-permeable cation channel of the TRPC } \\
\text { family ( } 854 \text { aa) }\end{array}$ & $\begin{array}{l}\text { MOs of spermatid } \\
\text { Cell body and } \\
\text { pseudopod of } \\
\text { sperm }\end{array}$ \\
\hline spe-42 & V & $\begin{array}{l}\text { Six-pass transmembrane protein with the } \\
\text { DC-STAMP and RING finger domains ( } 774 \mathrm{aa})\end{array}$ & ND \\
\hline
\end{tabular}

Chr. chromosome, $a a$ amino acid, $N D$ not determined 


\subsection{2 spe Genes Involved in Spermiogenesis}

\subsubsection{1 spe-8 Class-Dependent Spermiogenesis}

Mutants lacking either of the spe- 8 class genes all exhibit nearly the same phenotype: hermaphrodites are self-sterile and males are cross-fertile [spe- 8 (L'Hernault et al. 1988; Shakes and Ward 1989), spe-12 (L'Hernault et al. 1988; Shakes and Ward 1989; Nance et al. 1999), spe-19 (Geldziler et al. 2005), spe-27 (Minniti et al. 1996), and spe-29 (Nance et al. 2000)]. These mutant hermaphrodites, but not males, produce spermatids that do not undergo spermiogenesis in vivo.

Figure 18.4 shows one of the predicted models for $C$. elegans spermiogenesis (Nishimura and L'Hernault 2010). Spermatids derived from both hermaphrodites and males of spe- 8 class mutants exhibit normal cytology before and after in vitro activation with Monensin or TEA (L'Hernault 2009; Nishimura and L'Hernault 2010) (see Sect. 18.2.2). However, by in vitro treatment with Pronase, spe- 8 class mutant spermatids from either sex arrest at an intermediate stage; spiky projections are extended from mutant spermatids, but they never transform into pseudopods. Hence, spe- 8 class mutant spermatids are likely to have defects in the spermiogenesis pathway that is affected by Pronase. These data suggest that both hermaphrodite- and male-derived spermatids contain two pathways for activation into sperm, which are dependent on or independent of spe- 8 class genes. The spe- 8 classdependent pathway seems to act during spermiogenesis in hermaphrodites, whereas males probably utilize the spe- 8 class-independent pathway for spermatid activation. Among spe- 8 class genes, spe-12 might be required for both the spermiogenesis pathways because spe-12 mutant males show partially defective spermatid activation.

spe-6 and spe-4 are required in spermatocytes for proper complex formation of the MO and the fibrous body (FB), which is an assembly of MSP bundles (L'Hernault 2009; Nishimura and L'Hernault 2010). Intriguingly, both these two genes also act in spermiogenesis; there are non-null, suppressor alleles of spe-6 (Muhlrad and Ward 2002) and spe-4 (Gosney et al. 2008) that rescue the selfsterility of spe-8 class mutant hermaphrodites. Thereby, SPE-6 and SPE-4 (Table 18.1), which are a casein kinase 1-like Ser/Thr kinase and a presenilin 1-like aspartyl protease, respectively, appear to be downstream of the SPE-8 class proteins (Fig. 18.4). At present, how SPE-6 and SPE-4 function during spermiogenesis is controversial. One of the possible interpretations (Fig. 18.4) is that SPE-6 is active in resting spermatids to phosphorylate SPE-4, which contains predicted phosphorylation sites by casein kinase 1: this might disturb SPE-4 to appropriately cleave a membranous protein substrate(s), resulting in blocking spermatid activation. During spermiogenesis, SPE-6 activity is conversely reduced, and dephosphorylated SPE-4 becomes capable of proteolytically splitting its substrate(s). 


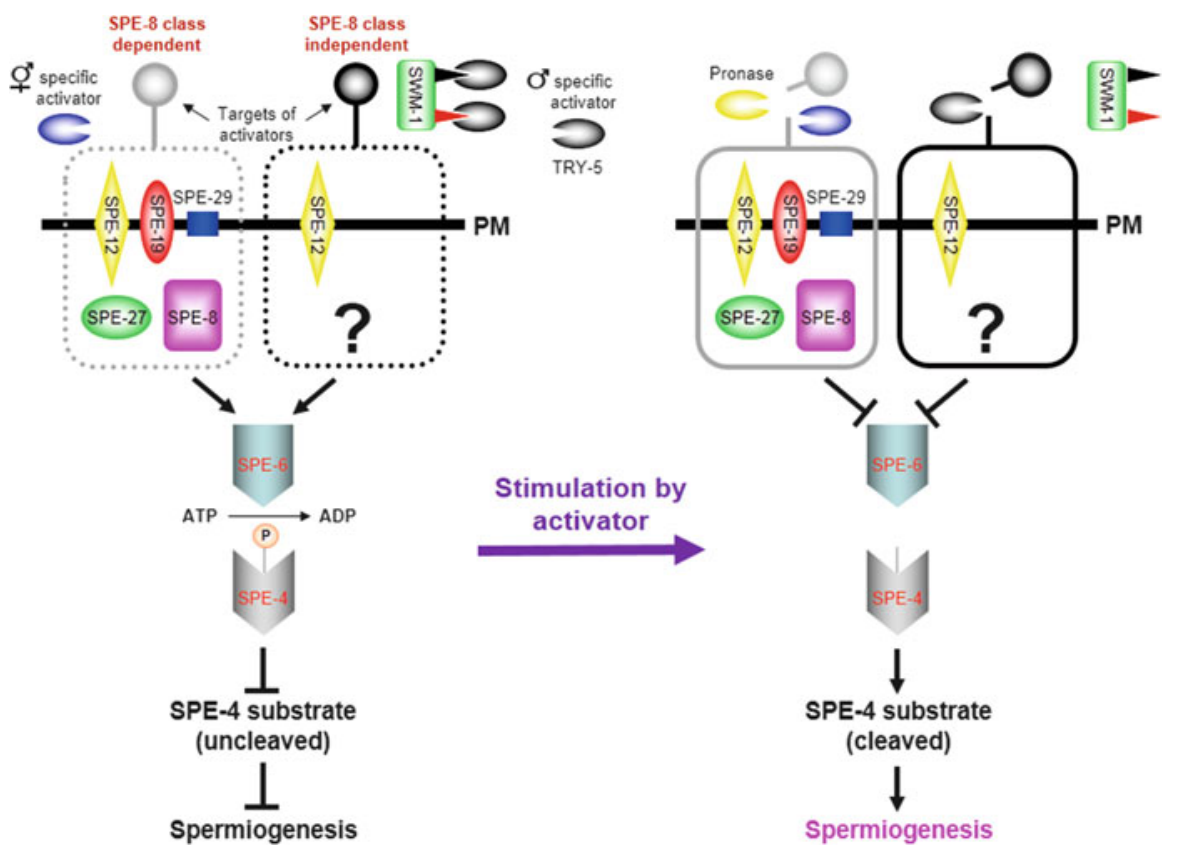

Fig. 18.4 Two predicted pathways for C. elegans spermiogenesis. To regulate spermiogenesis, there are likely two distinct pathways in all wild-type spermatids from hermaphrodites and males: one pathway is spe- 8 class dependent and another is spe- 8 class independent. Sex-specific activators probably determine which pathway is utilized. The spe- 8 class-dependent pathway appears to be stimulated by an unknown hermaphrodite-derived activator(s) or the serine protease mixture Pronase. For males, the serine protease TRY- 5 presumably activates spermatids via the spe- 8 classindependent pathway, and its protease activity is blocked by the trypsin inhibitor-like protein SWM-1. To initiate spermiogenesis, these activators might be required to cleave a certain cellsurface protein(s). SPE-6, a casein kinase 1-like Ser/Thr kinase, is downstream of SPE-8 class proteins and is one of the common points between these two pathways. This kinase perhaps phosphorylates (shown as "P" in a ball) the presenilin 1-like aspartyl protease SPE-4, because SPE-4 has predicted phosphorylation sites by casein kinase 1 . Then, by the phosphorylation, SPE-4 may become incapable of splitting a membranous protein substrate(s), leading to blocking spermiogenesis. On the other hand, SPE-6 activity presumably requires to be reduced so that dephosphorylated SPE-4 can cleave its substrate(s) for onset of spermiogenesis. Note that other interpretations for how SPE-6 and SPE-4 act downstream of SPE-8 class proteins are likely available. In this figure, the active and inactive status of each pathway are shown by solid and broken lines, respectively. Thick black arrows represent positive regulation, whereas negative regulation is expressed by $T$-shaped lines. $P M$ plasma membrane. (These figures were taken from the article by Nishimura and L'Hernault 2010 with the publisher's permission)

\subsubsection{2 spe-8 Class-Independent Spermiogenesis}

As described in Sect. 18.2.2, try-5 encodes a soluble serine protease that is a component of the seminal fluids (Smith and Stanfield 2011) (Table 18.1). Hermaphrodites and males of try-5 mutants are both fertile. However, the seminal fluids of try-5 
mutant males are incompetent to activate spermatids in vivo, unlike those of wild-type males. Moreover, males carrying double mutations in try-5 and spe-27 or spe-29 are sterile, whereas male fertility is not affected by any single mutation in spe-27, spe-29, or try-5. Therefore, it is postulated from these findings that (1) TRY-5 protease probably activates male-derived spermatids via the spe- 8 classindependent pathway, and (2) male-derived spermatids can respond to a hermaphrodite-produced activator(s) that stimulates the spe- 8 class-dependent pathway.

swm-1 is also implicated in male fertility (Stanfield and Villeneuve 2006; Smith and Stanfield 2011). In swm-1 mutant males, spermatids are ectopically activated into sperm within the male gonads, and these mutant sperm are not transferred normally into hermaphrodites during mating. Because swm- 1 mutant sperm are capable of fertilizing oocytes after artificial insemination of the mutant sperm into wild-type hermaphrodites, the sterility of $s w m-1$ mutant males is probably caused by this transfer defect. The $s w m-1$ gene encodes a soluble protein with two trypsin inhibitor-like domains (Table 18.1), both of which seem to react with TRY-5 and/or another serine protease(s) that is related to TRY-5 (ex. activator or substrate of TRY5) (Smith and Stanfield 2011). Thus, SWM-1 is very likely to block the premature spermatid activation by TRY-5 within the seminal vesicles. After ejaculation, TRY-5 or its related serine protease(s) probably become free from SWM-1 in the uterus, and activation of male spermatids immediately occurs.

Recently, it was found that male spermatids of Ascaris suum (As) secrete the Serpin (serine protease inhibitor) family protein As_SRP-1, which seems to block the activity of As_TRY-5 (Ascaris orthologue of TRY-5) (Zhao et al. 2012). Therefore, C. elegans SPR-1 (Table 18.1), in addition to SWM-1, presumably regulates the TRY-5 protease activity to prevent male spermatids from ectopic activation in the male reproductive tract.

\subsection{3 spe Genes Involved in Fertilization}

As one of the spe-9 class genes is disturbed, hermaphrodites and males produce otherwise normal sperm that cannot fertilize oocytes [spe-9 (L'Hernault et al. 1988; Singson et al. 1998; Zannoni et al. 2003; Putiri et al. 2004), spe-13 (L'Hernault et al. 1988; Putiri et al. 2004), spe-38 (Chatterjee et al. 2005), spe-41/trp-3 (Xu and Sternberg 2003), and spe-42 (Kroft et al. 2005)]. There are at least two interpretations for this phenotype. First, spe-9 class mutant sperm bind to the oocyte plasma membrane with very low affinities and thus mutant sperm are easily detached from the oocyte plasma membrane or cannot proceed to the next step, such as spermoocyte fusion. Second, spe- 9 class mutant sperm normally bind to the oocyte plasma membrane, but a defect in fusion occurs.

At any rate, it is very likely that SPE-9 class proteins (Fig. 18.5 and Table 18.2) play critical roles during sperm-oocyte interactions (binding or fusion). 
a SPE-9

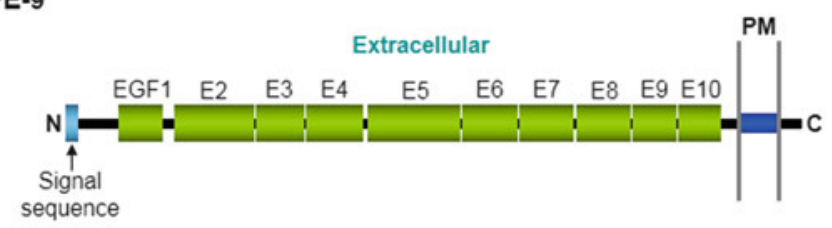

b SPE-42
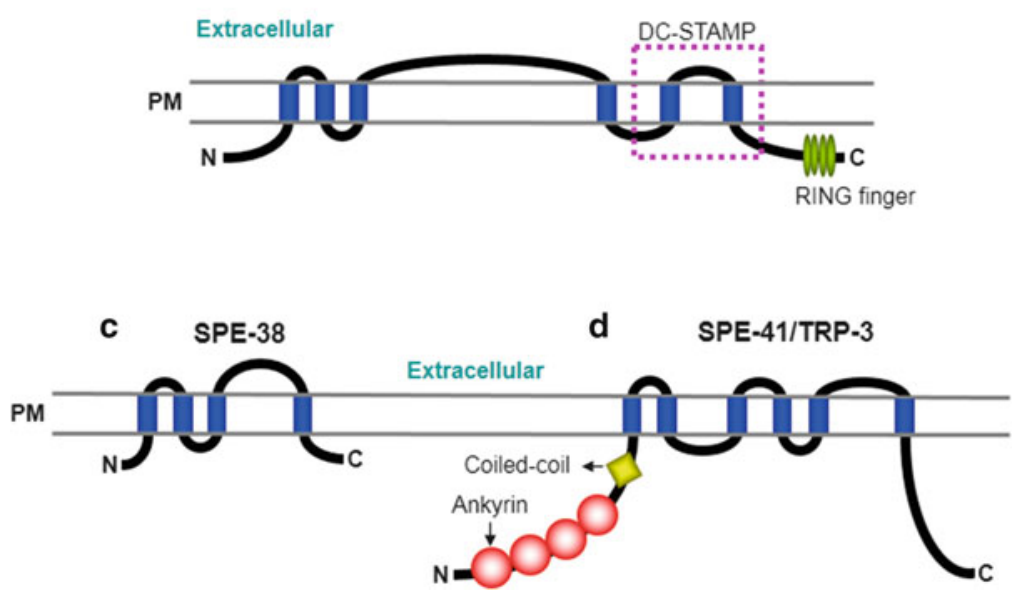

Fig. 18.5 SPE-9 class proteins presumably required for sperm-oocyte interactions. (a) SPE-9. E, EGF-like domain. (b) SPE-42. The region corresponding to the DC-STAMP domain is indicated by a broken line. (c) SPE-38. (d) SPE-41/TRP-3. In these figures, cylinders shown in the plasma membrane $(P M)$ represent the transmembrane domains. $N \mathrm{~N}$-terminus, $C \mathrm{C}$-terminus. (These figures were taken from the article by Nishimura and L'Hernault 2010 with the publisher's permission)

\subsubsection{1 spe-9}

SPE-9 is a single-pass transmembrane protein containing ten epidermal growth factor (EGF)-like domains (Fig. 18.5a). The overall domain structure of SPE-9 is similar to those of ligands for the Notch/LIN-12/GLP-1 family: Delta, Serrate, and Jagged1. Moreover, as these Notch family ligand proteins, the EGF-like domains of SPE-9 are essential for its function in C. elegans fertilization (Putiri et al. 2004). Therefore, SPE-9 might be a ligand for a putative sperm receptor(s) on the oocyte surface, although SPE-9 has no DSL domain, which characterizes the Delta/Serrate/ LAG-2 family (Cordle et al. 2008). This hypothesis does not conflict with the fact that SPE-9 is localized on the surface of pseudopods, through which sperm bind/ fuse with oocytes. 


\subsubsection{2 spe-42}

SPE-42 is a six-pass transmembrane protein (Fig. 18.5b), but its localization is yet unclear. This protein contains two functional domains, the DC-STAMP (dendritic cell-specific transmembrane protein) and the C4C4-type RING finger domains (Fig. 18.5b). Mammalian DC-STAMPs are known to mediate cell-cell fusion of osteoclasts and foreign-body giant cells. Moreover, the Drosophila gene sneaky, presumably the orthologue of spe-42, is involved in sperm plasma membrane breakdown after the fly sperm enter the oocytes (Wilson et al. 2006). Thus, SPE-42 might act in sperm-oocyte fusion via the DC-STAMP domain.

The C-terminal RING finger domain of SPE-42 is essentially required for C. elegans fertilization (Wilson et al. 2011). The C4C4-type RING finger domains generally act in protein-protein interactions, suggesting that this domain of SPE-42 could be a binding site with another sperm protein(s). It is also possible that the SPE-42 RING finger domain might have a ubiquitin E3 ligase activity, similar to the human RING finger protein CNOT4, although most RING finger E3 ligases are a C3HC4 type. Consequently, SPE-42 possibly catalyzes the ubiquitination of a sperm protein(s) that acts during sperm-oocyte interactions to regulate the localization or function of that protein(s) (Nishimura and L'Hernault 2010).

\subsubsection{3 spe-38}

This gene encodes a four-pass transmembrane protein with no other significant domains (Fig. 18.5c). SPE-38 is located within the MOs in spermatids, but it appears on the cell surface after MO fusion with the spermatid plasma membrane. SPE-38 is probably involved in sperm-oocyte interactions because it localizes to pseudopods as does SPE-9. Recently, this protein was found to associate with SPE-41/ TRP-3 to regulate the SPE-41/TRP-3 localization (Singaravelu et al. 2012).

\subsubsection{4 spe-41/trp-3}

SPE-41/TRP-3 belongs to the TRPC [transient receptor potential (TRP)-canonical] superfamily of cation channels (Fig. 18.5d). Indeed, SPE-41/TRP-3 was demonstrated to act as a calcium channel in sperm, but not in spermatids. This finding is in good agreement with the SPE-41/TRP-3 localization; SPE-41/TRP-3 is intracellularly localized in the MOs of spermatids, and during spermiogenesis, this protein translocates onto the surface of both the pseudopod and the cell body. Again, the pseudopod is the place where sperm-oocyte interactions are thought to occur. On the $C$. elegans sperm surface, SPE-41/TRP-3 probably forms a homo- or heterotetramer through the ankyrin and coiled-coil domains, as do other members of the TRP family. Sperm-oocyte binding might produce a signal to open the SPE-41/TRP-3 channel, and the ensuing calcium influx would trigger gamete fusion. 
Moreover, we have recently found that a male germline-specific gene encoding an immunoglobulin (Ig)-like transmembrane protein is essentially required for C. elegans fertilization, similar to the mouse Izumol gene (Inoue et al. 2005). This observation indicates that the C. elegans Ig-like gene belongs to the spe-9 class, and functional studies of this gene are currently ongoing in our laboratories.

\subsection{Perspectives}

We see two major reasons why $C$. elegans is useful for the study of reproductive biology. First, as already described, numerous mutants that are defective in reproduction can be easily created. These mutants are powerful tools to identify C. elegans genes that are indispensable for reproduction. For example, one area that is especially important and poorly understood in any species is the mechanism of sperm-oocyte binding and fusion. C. elegans spe-9 class mutants, in which otherwise normal sperm fail to properly bind to or fuse with the oocyte plasma membrane, are the largest collection of gamete interaction-defective mutants in any organism.

Second, C. elegans is optically transparent, which allows in vivo analysis of reproduction. Because small chemicals, peptides, and antibodies can be introduced into worms by soaking, feeding, or microinjection, this animal can be used as a kind of "in vivo test tube," which is a unique property in reproductive research. These experiments might result in development and evaluation of drugs for infertile or contraceptive therapies.

A current problem of the C. elegans reproductive research is that an IVF system is not available (see Sect. 18.2.3). Development of this system enables us to determine the precise point at which mutants affect fertilization. Moreover, an IVF assay would be useful to examine candidate drugs for infertile or contraceptive therapy in addition to testing them by in vivo C. elegans fertilization.

Acknowledgments These studies were supported by grants from the National Science Foundation (to S.W.L., IOB-0544180) and National Institute of Health (to S.W.L., GM082932), USA, and by Grant-in-aid for Scientific Research on Innovative Areas from the MEXT (to H.N., 24112716), Japan. We thank all past and current members of the L'Hernault lab and the Nishimura lab for valuable discussions and suggestions.

Open Access: This article is distributed under the terms of the Creative Commons Attribution Noncommercial License which permits any noncommercial use, distribution, and reproduction in any medium, provided the original author(s) and source are credited. 


\section{References}

C. elegans Sequencing Consortium (1998) Genome sequence of the nematode C. elegans: a platform for investigating biology. Science 282:2012-2018. doi:10.1126/science.282.5396. 2012

Chatterjee I, Richmond A, Putiri E, Shakes DC, Singson A (2005) The Caenorhabditis elegans spe-38 gene encodes a novel four-pass integral membrane protein required for sperm function at fertilization. Development (Camb) 132:2795-2808

Cordle J, Johnson S, Tay JZ, Roversi P, Wilkin MB, de Madrid BH, Shimizu H, Jensen S, Whiteman P, Jin B, Redfield C, Baron M, Lea SM, Handford PA (2008) A conserved face of the Jagged/ Serrate DSL domain is involved in Notch trans-activation and cis-inhibition. Nat Struct Mol Biol 15:849-857. doi:10.1038/nsmb.1457

Geldziler B, Chatterjee I, Singson A (2005) The genetic and molecular analysis of spe-19, a gene required for sperm activation in Caenorhabditis elegans. Dev Biol 283:424-436

Gosney R, Liau WS, LaMunyon CW (2008) A novel function for the presenilin family member spe-4: inhibition of spermatid activation in Caenorhabditis elegans. BMC Dev Biol 8:44. doi:10.1186/1471-213X-8-44

Inoue N, Ikawa M, Isotani A, Okabe M (2005) The immunoglobulin superfamily protein Izumo is required for sperm to fuse with eggs. Nature (Lond) 434:234-238

Kroft TL, Gleason EJ, L'Hernault SW (2005) The spe-42 gene is required for sperm-egg interactions during C. elegans fertilization and encodes a sperm-specific transmembrane protein. Dev Biol 286:169-181

L'Hernault SW (2009) The genetics and cell biology of spermatogenesis in the nematode C. elegans. Mol Cell Endocrinol 306:59-65. doi:0.1016/j.mce.2009.01.008

L'Hernault SW, Shakes DC, Ward S (1988) Developmental genetics of chromosome I spermatogenesis-defective mutants in the nematode Caenorhabditis elegans. Genetics 120: $435-452$

Minniti AN, Sadler C, Ward S (1996) Genetic and molecular analysis of spe-27, a gene required for spermiogenesis in Caenorhabditis elegans hermaphrodites. Genetics 143:213-223

Muhlrad PJ, Ward S (2002) Spermiogenesis initiation in Caenorhabditis elegans involves a casein kinase 1 encoded by the spe-6 gene. Genetics 161:143-155

Nance J, Minniti AN, Sadler C, Ward S (1999) spe-12 encodes a sperm cell surface protein that promotes spermiogenesis in Caenorhabditis elegans. Genetics 152:209-220

Nance J, Davis EB, Ward S (2000) spe-29 encodes a small predicted membrane protein required for the initiation of sperm activation in Caenorhabditis elegans. Genetics 156:1623-1633

Nishimura H, L'Hernault SW (2010) Spermatogenesis-defective (spe) mutants of the nematode Caenorhabditis elegans provide clues to solve the puzzle of male germline functions during reproduction. Dev Dyn 239:1502-1514. doi:10.1002/dvdy.22271

Putiri E, Zannoni S, Kadandale P, Singson A (2004) Functional domains and temperature-sensitive mutations in SPE-9, an EGF repeat-containing protein required for fertility in Caenorhabditis elegans. Dev Biol 272:448-459

Shakes DC, Ward S (1989) Initiation of spermiogenesis in C. elegans: a pharmacological and genetic analysis. Dev Biol 134:189-200

Singaravelu G, Chatterjee I, Rahimi S, Druzhinina MK, Kang L, Xu XZ (2012) Singson A (2012) The sperm surface localization of the TRP-3/SPE-41 $\mathrm{Ca}^{2+}$-permeable channel depends on SPE38 function in Caenorhabditis elegans. Dev Biol 365:376-383. doi:10.1016/j.ydbio.2012.02.037

Singson A, Mercer KB, L'Hernault SW (1998) The C. elegans spe-9 gene encodes a sperm transmembrane protein that contains EGF-like repeats and is required for fertilization. Cell 93:71-79

Singson A, Hang JS, Parry JM (2008) Genes required for the common miracle of fertilization in Caenorhabditis elegans. Int J Dev Biol 52:647-656. doi:10.1387/ijdb.072512as

Smith JR, Stanfield GM (2011) TRY-5 is a sperm-activating protease in Caenorhabditis elegans seminal fluid. PLoS Genet 7:e1002375. doi:10.1371/journal.pgen.1002375 
Stanfield GM, Villeneuve AM (2006) Regulation of sperm activation by SWM-1 is required for reproductive success of $C$. elegans males. Curr Biol 16:252-263

Wilson KL, Fitch KR, Bafus BT, Wakimoto BT (2006) Sperm plasma membrane breakdown during Drosophila fertilization requires sneaky, an acrosomal membrane protein. Development (Camb) 133:4871-4879

Wilson LD, Sackett JM, Mieczkowski BD, Richie AL, Thoemke K, Rumbley JN, Kroft TL (2011) Fertilization in C. elegans requires an intact C-terminal RING finger in sperm protein SPE-42. BMC Dev Biol 11:10

Xu XZ, Sternberg PW (2003) A C. elegans sperm TRP protein required for sperm-egg interactions during fertilization. Cell 114:285-297

Zannoni S, L'Hernault SW, Singson AW (2003) Dynamic localization of SPE-9 in sperm: a protein required for sperm-oocyte interactions in Caenorhabditis elegans. BMC Dev Biol 3:10

Zhao Y, Sun W, Zhang P, Chi H, Zhang MJ, Song CQ, Ma X, Shang Y, Wang B, Hu Y, Hao Z, Hühmer AF, Meng F, L'Hernault SW, He SM, Dong MQ, Miao L (2012) Nematode sperm maturation triggered by protease involves sperm-secreted serine protease inhibitor (serpin). Proc Natl Acad Sci USA 109:1542-1547. doi:10.1073/pnas.1109912109 